\title{
Diabetic Neuropathy: Emerging Victory of Antioxidants
}

\section{Ivan M Petyaev and Yuriy K Bashmakov*}

Lycotec Ltd, Granta Park, Cambridge, CB21 6GP, United Kingdom

Type 2 Diabetes Mellitus (T2DM) is a systemic metabolic disorder associated with serious and often life-threatening complications. Diabetic neuropathy is a common complication affecting more than half of T2DM patients [1]. Neurological abnormalities affecting the distal lower extremities are known to appear in the early stages of $\mathrm{T} 2 \mathrm{DM}$ and even in patients with pre-diabetes and abnormal glucose tolerance [2].

There is no widely accepted consensus on the treatment of neurological abnormalities in diabetics. No drugs have been specifically approved for patients with diabetic neuropathy. For decades pharmacological interventions aimed at the normalization of glucose turnover and hemoglobin A (1C) level have remained a mainstay of therapy for diabetic neuropathy patients [3]. Other pharmacological options include pain management (tricyclic antidepressants, serotoninnorepinephrine reuptake inhibitors, $a 2 \delta$ ligands, antiepileptics, opioids) and anti-inflammatory drugs [4]. Although innovative treatment approaches (gene therapy, steam cell therapy, use of neurotrophic and growth factors) are under development, their rapid introduction into routine clinical practice seems to be problematical [4,5]. Even though symptomatic treatment usually produces some temporary improvements in the health status of patients, the etiology- and mechanism-driven approach to the treatment of diabetic neuropathy is not available, remaining in its infancy due to lack of precise knowledge about the pathophysiological mechanisms underlying neurological defects in T2DM. It has been clearly shown for decades that diabetic neuropathy is a direct consequence of abnormal glucose turnover mirroring improvements and deteriorations in glucose homeostasis. Chronic hyperglycemia in T2DM leads to up to a four-fold increase in the neuronal glucose level which cannot be neutralized by anaerobic and glycolytic burst as happens in the muscle cells [6]. Glucose neurotoxicity develops due to the unique oxidative biochemistry of glucose leading to accumulation of free radicals in the neuronal tissue via non-enzymatic, enzymatic and mitochondrial mechanisms [7]. Hyperglycemia promotes the formation of $\mathrm{O}_{2}$ - superoxide through the polyol (sorbitol) pathway which is accompanied by non-enzymatic generation of Amadori products and the subsequent formation of advanced glycation end products [8]. Moreover, reactive oxygen species have been shown to be generated in the nitric oxide synthase, $\mathrm{NAD}(\mathrm{P}) \mathrm{H}$ oxidase and xanthine oxidase systems [9]. It is now believed that oxidative damage is a primary pathogenetic mechanism causing impaired axonal transport, structural breakdown of Schwann cells and subsequent abnormalities in the propagation of action potential [10].

Due to advances in the understanding of the mechanisms of oxidative stress it recently became feasible to target oxidative stress using in particular nutraceuticals with antioxidant properties. This may create an important novel strategy in the treatment of diabetic neuropathy. The latest developments in clinical and experimental diabetology fully support this assumption. The most exciting results come from the recently published multicenter randomized doublemasked parallel Neurological Assessment of Thioctic Acid in Diabetic Neuropathy (NATHAN) 1 trial which involved 460 patients with mild to moderate distal symmetric diabetic polyneuropathy. It was well documented that four year treatment with $\alpha$-lipoic acid led to clinically relevant improvement of neurological functions as evaluated by seven nerve function tests in patients with diabetic polyneuropathy [11] Although $\alpha$-lipoic acid treatment did not affect the nerve conduction parameters in patients, the Neuropathy Impairment Score of the lower limbs (NIS-LL) was improved in the lipoic acid treatment group and worsened in the placebo [11]. Similar improvements in neurological functions were recently shown on a smaller scale in comparable clinical settings [12] and animal studies [13,14]. Therefore, despite of some limitations, the results of NATHAN-1 trial represent the first systematic and considerable piece of evidence revealing the therapeutic effect of an antioxidant in diabetic neuropathy confirmed by others. Such an outcome is particularly welcome due to multiple failures to show any clinically measurable effect of antioxidants in different clinical settings. a -lipoic acid, otherwise known as thioctic acid, is an orthomolecular nutrient with extremely potent free radical scavenging capabilities reported in different systems [15]. Besides reducing lipid peroxidation, a -lipoic acid has been shown to normalize microcirculation and insulin sensitivity, enhance endogenous superoxide dismutase, reduce ischemia-reperfusion syndrome and prevent apoptosis [16]. Generally, $a$-lipoic acid is well tolerated and has low toxicity. However, the results of the NATHAN 1 trial reveal a tendency towards a higher incidence of adverse effects in the treatment group as compared to the placebo. This issue could be addressed in the future by a lowering of the therapeutic dose or the use of other isoforms of the compound. The antioxidant properties of $\alpha$-lipoic acid are mediated by balanced conversion of the compound to dihydrolipoic acid, a reduced metabolite [15]. It has been shown recently under in vitro conditions that dihydrolipoic acid has superior antioxidant potency when compared to a -lipoic acid [17]. Therefore assessment of in vitro and in vivo toxicity and the possible introduction of dihydrolipoic acid into therapeutic formulations may provide a reasonable solution for the reduction of adverse effects of lipoic acid.

On the other hand, the implications of the NATHAN 1 trial go far beyond the therapeutic use of $\alpha$-lipoic acid in diabetes care. It has been shown recently that the effect of $\alpha$-lipoic acid in diabetic neuropathy can be enhanced by other antioxidants, in particular superoxide dismutase [18]. Therefore it is plausible to assume that the effect of $\alpha$-lipoic acid is unlikely to be attributable to its particular chemical nature but is rather related to the antioxidant properties of the compound. Indeed, many other nutrients (resveratrol, lycopene, soy isoflavones) with antioxidant properties have been shown in both in vitro and in vivo systems to have a significant positive impact on the complications of T2DM [19]. The success of the NATHAN 1 trial has opened the door for

*Corresponding author: Yuriy K Bashmakov, Scientific Officer, Lycotec Ltd, Granta Park, Cambridge, Cambridgeshire, United Kingdom, Tel: +44 1223420 721; Fax: +44 1223424 516; E-mail: yuriy.bashmakov@lycotec.com

Received February 28, 2012; Accepted April 16, 2012; Published April 20, 2012

Citation: Petyaev IM, Bashmakov YK (2012) Diabetic Neuropathy: Emerging Victory of Antioxidants. J Diabetes Metab S5:e001. doi:10.4172/2155-6156.S5e001

Copyright: (C) 2012 Petyaev IM, et al. This is an open-access article distributed under the terms of the Creative Commons Attribution License, which permits unrestricted use, distribution, and reproduction in any medium, provided the original author and source are credited. 
new clinical trials which may unveil the possible benefits of antioxidant use in T2DM. However, there are multiple challenges to clinical use of nutrients with antioxidant properties. Unlike a -lipoic acid many of them are susceptible to auto-oxidation [20], have limited absorption [21] and/or can be rapidly metabolized by the gut microbiota [22] Therefore stabilized formulations of antioxidants would be required for new clinical research projects.

Nevertheless, the success of the NATHAN 1 trial gives great inspiration to new attempts at micronutrient use in diabetes. It is important to realize that T2DM is a nutritional disorder arising entirely from excessive and/or unbalanced food intake. Therefore nutritional interventions as well as the selective use of different food ingredients seem to be a most appropriate and promising treatment option for T2DM. The idea of using food ingredients as medicinal agents originated in ancient times. In particular, Hippocrates' statement "Let food is your medicine and medicine is your food" provides a backbone for the current philosophy of T2DM management. The diverse and extremely powerful physiological effects of some food constituents, comparable to the effects of some pharmacological agents - has led to the introduction of the relatively new term 'nutraceuticals' whose potential therapeutic use in T2DM patients has yet to be further explored in well-designed clinical trials.

\section{References}

1. Vincent AM, Callaghan BC, Smith AL, Feldman EL (2011) Diabetic neuropathy: cellular mechanisms as therapeutic targets. Nat Rev Neurol 7: 573-583.

2. Basić-Kes V, Zavoreo I, Rotim K, Bornstein N, Rundek T, et al. (2011) Recommendations for diabetic polyneuropathy treatment. Acta Clin Croat 50: 289-302.

3. Ziegler D (2011) Current concepts in the management of diabetic polyneuropathy. Curr Diabetes Rev 7: 208-220.

4. Smith HS, Argoff CE (2011) Pharmacological treatment of diabetic neuropathic pain. Drugs 71: 557-589.

5. Kim H, Kim JJ, Yoon YS (2012) Emerging therapy for diabetic neuropathy: Cell therapy targeting vessels and nerves. Endocr Metab Immune Disord Drug Targets [Epub ahead of print]

6. Tomlinson DR, Gardiner NJ (2008) Glucose neurotoxicity. Nat Rev Neurosci 9: $36-45$.

7. Golbidi S, Badran M, Laher I (2011) Diabetes and alpha lipoic Acid. Fron Pharmacol 2: 69.
8. Miranda-Massari JR, Gonzalez MJ, Jimenez FJ, Allende-Vigo MZ, Duconge $J$ (2011) Metabolic correction in the management of diabetic periphera neuropathy: improving clinical results beyond symptom control. Curr Clin Pharmacol 6: 260-273

9. Yamagishi S, Matsui T (2011) Nitric oxide, a janus-faced therapeutic target for diabetic microangiopathy-Friend or foe? Pharmacol Res 64: 187-194

10. Firuzi O, Miri R, Tavakkoli M, Saso L (2011) Antioxidant therapy: current status and future prospects. Curr Med Chem 18: 3871-3888

11. Ziegler D, Low PA, Litchy WJ, Boulton AJ, Vinik Al, et al. (2011) Efficacy and safety of antioxidant treatment with $\alpha$-lipoic acid over 4 years in diabetic polyneuropathy: the NATHAN 1 trial. Diabetes Care 34: 2054-2060.

12. Mijnhout GS, Kollen BJ, Alkhalaf A, Kleefstra N, Bilo HJG (2012) Alpha lipoic Acid for symptomatic peripheral neuropathy in patients with diabetes: a metaanalysis of randomized controlled trials. Int J Endocrinol: 456279

13. Morgado C, Pereira-Terra P, Tavares I (2011) a-Lipoic acid normalizes nociceptive neuronal activity at the spinal cord of diabetic rats. Diabetes Obes Metab 13: 736-741

14. Skalská S, Kucera P, Goldenberg Z, Stefek M, Kyselová Z, et al. (2010) Neuropathy in a rat model of mild diabetes induced by multiple low doses of streptozotocin: effects of the antioxidant stobadine in comparison with a highdose alpha-lipoic acid treatment. Gen Physiol Biophys 29: 50-58.

15. Goraca A, Huk-Kolega H, Piechota A, Kleniewska P, Ciejka E, et al. (2011) Lipoic acid - biological activity and therapeutic potential. Pharmacol Rep 63 849-858.

16. Papanas N, Maltezos E (2012) a-Lipoic acid, diabetic neuropathy, and Nathan's prophecy. Angiology 63: 81-83.

17. Ghibu S, Lauzier B, Delemasure S, Amoureux S, Sicard P, et al. (2009) Antioxidant properties of alpha-lipoic acid: effects on red blood membrane permeability and adaptation of isolated rat heart to reversible ischemia. Mo Cell Biochem 320: 141-148.

18. Bertolotto F, Massone A (2012) Combination of Alpha Lipoic Acid and Superoxide Dismutase Leads to Physiological and Symptomatic Improvements in Diabetic Neuropathy. Drugs R D 12: 29-34.

19. Golbidi S, Ebadi SA, Laher I (2011) Antioxidants in the treatment of diabetes. Curr Diabetes Rev 7: 106-125.

20. Xianquan S, Shi J, Kakuda Y, Yueming J (2005) Stability of lycopene during food processing and storage. J Med Food 8: 413-422.

21. Kwon DY, Daily JW 3rd, Kim HJ, Park S (2010) Antidiabetic effects of fermented soybean products on type 2 diabetes. Nutr Res 30: 1-13.

22. Selma MV, Espín JC, Tomás-Barberán FA (2009) Interaction between phenolics and gut microbiota: role in human health. J Agric Food Chem 57 6485-6501. 